\title{
Energie marémotrice et environnement aquatique
}

\author{
Ch. Rétière \\ Laboratoire maritime - Muséum d'histoire naturelle - Dinard
}

Le bassin de La Rance sur la côte nord de Bretagne constitue l'unique site où a été évalué en vraie grandeur et après 20 années de fonctionnement l'impact écologique d'un aménagement marémoteur. La période d'isolement de l'estuaire durant la construction fut particulièrement dommageable pour l'environnement. Progressivement, après la mise en service, une flore et une faune, de plus en plus diversifiées, se sont établies. Leurs modes de répartition, de groupements en unités cénotiques et la nature des relations qu'elles entretiennent entre elles, témoignent, à des degrés divers, d'un ajustement biologique aux nouvelles conditions environnementales. L'ouvrage marémoteur dont la perméabilité biologique a permis la recolonisation du bassin laisse également passer, à travers vannes et turbines, les organismes migrateurs. Le nouvel équilibre écologique, instauré au terme d'une dizaine d'années, demeure cependant fragile; lié à la stabilité des conditions abiotiques, il dépend pour une large part du mode d'exploitation de l'usine.

\section{Introduction}

L'idée d'exploiter l'énergie des marées est fort ancienne. Dès le $11^{\mathrm{e}}$ siècle on utilisait des moulins à marées sur les côtes atlantiques de France, d'Espagne et de GrandeBretagne. L'énergie récupérée suffisait à faire marcher des pompes et à moudre le blé.

De nos jours pour songer raisonnablement à exploiter ce type d'énergie la condition première est de disposer de sites où l'amplitude de la marée est forte; à l'échelle planétaire ils sont au nombre d'une vingtaine soumis à un marnage qui avoisine ou dépasse 10 mètres.

Aussi des projets marémoteurs se sont-ils faits jour à travers le monde: golfe de Gambay en Inde, baie de Garolim en Corée du Sud, baie de Fundy au Canada, estuaire de La Severn en Grande-Bretagne...

Devant l'ampleur des aménagements envisagés, certains chercheurs ont tenté d'en prévoir les effets sur l'environnement marin (MARTIN, 1970 ; GORDON et LONGHURST, 1979 ; SHAw, 1980 ; LARSEN et TOPINKA, 1984...). Mais à cet égard le bassin de La Rance, sur les côtes armoricaines de La Manche, constitue l'unique site où peut être évalué en vraie grandeur et au terme de vingt années de fonctionnement l'impact écologique d'un aménagement marémoteur.

\section{Tidal power and the aquatic environment}

The Rance basin on the northern coast of Brittany is the only site where a full-scale evaluation of the ecological impact of a tidal power scheme has been made, after 20 years of operation. The period of isolation of the estuary during the construction phase was particularly damaging for the environment. Gradually, after the scheme was put into service, an increasingly diverse flora and fauna became established. The patterns of distribution of this flora and fauna, their groupings into cenotic units and the nature of their inter-relationships, indicate a variable degree of biological adjustment to the new environmental conditions. The tidal power scheme, whose biological permeability has enabled re-colonisation of the basin to take place, also allows migratory organisms to pass via sluice gates and turbines. However, the new ecological equilibrium established in the space of 10 years remains fragile and, being linked to the stability of abiotic conditions, depends to a large extent on the operating conditions of the tidal power scheme. 


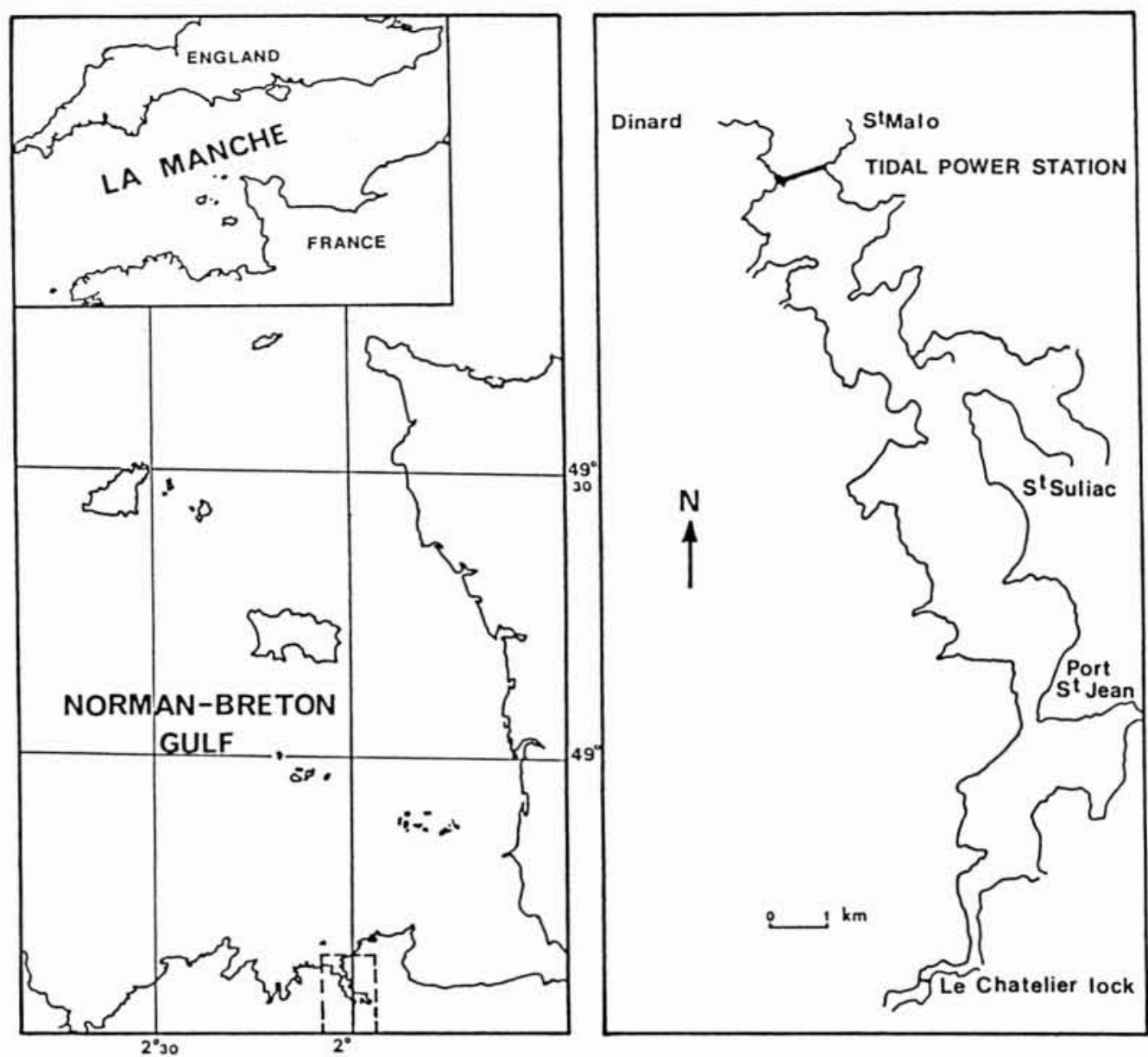

1. Le golfe normand-breton et l'estuaire de la Rance.

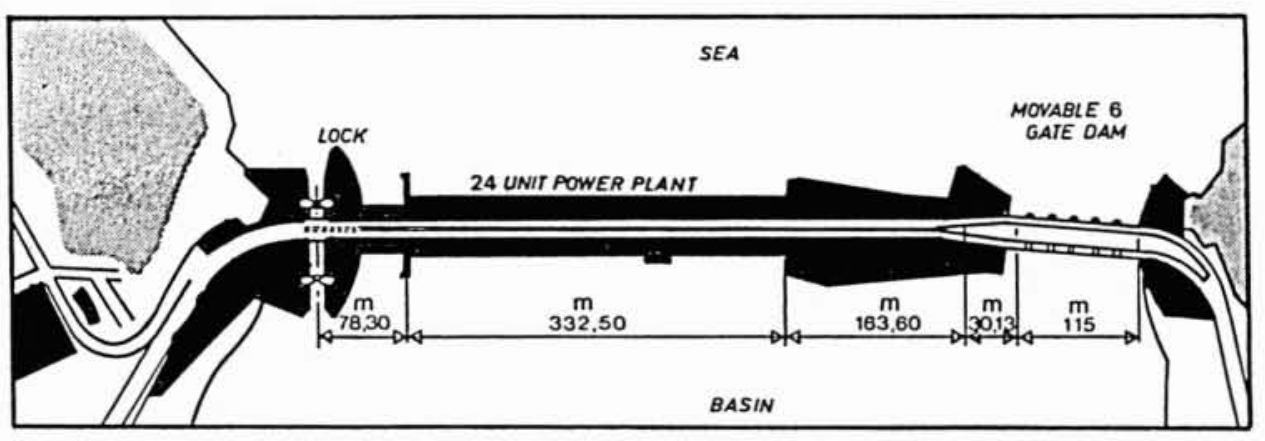

2. Le barrage et l'usine marémotrice.

Lock : Ecluse/24 Unit Power Plant : Usine proprement dite avec ses 24 groupes «bulbe »/Movable 6 Gate Dam : Barrage mobile formé de 6 vannes/Sea : Mer/Basin: Bassin. 


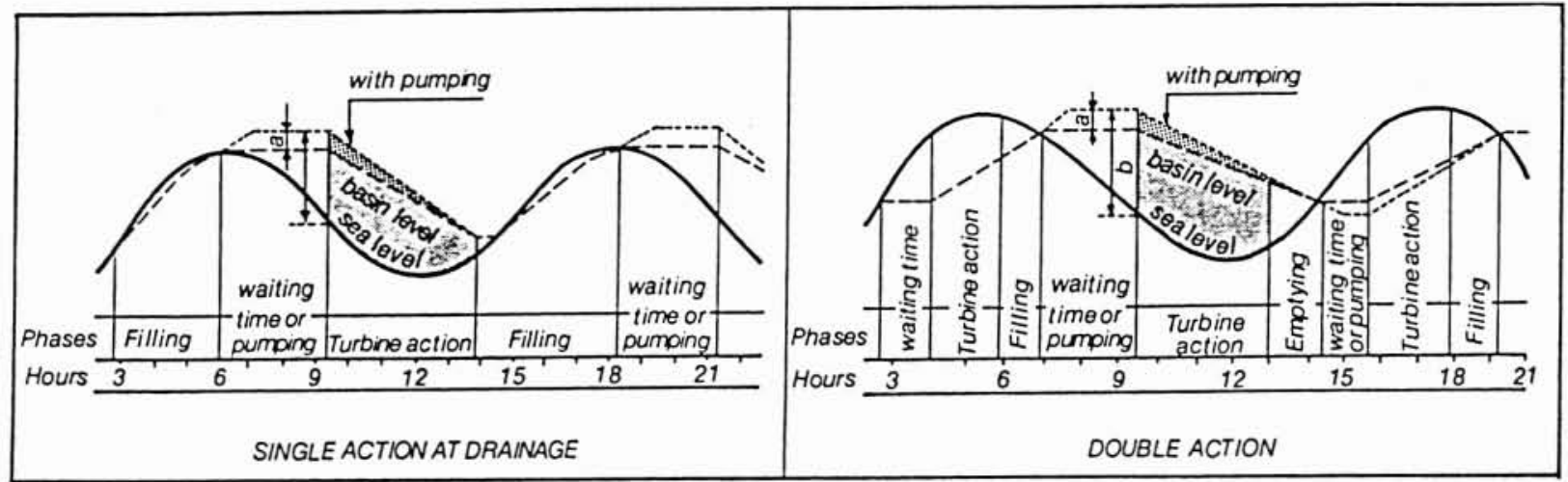

3. Courbes de marée comparées avec les variations de niveau du plan d'eau à l'intérieur du bassin (simple et double effets). (D'après Electricité de France.)

With pumping: avec pompage/Phases: phases/Hours: heures/Filling: remplissage/Waiting time : attente/Pumping: pompage/Turbine action: turbinage/Emptying: vidage/Basin level: niveau bassin/Sea level : niveau mer/Double action: double effet/Single action at drainage : simple effet au vidage.

Parmi les mers du nord-ouest de l'Europe, La Manche se singularise par le caractère mégatidal de ses marées, spécialement dans sa partie occidentale: l'amplitude en vive-eau d'équinoxe atteint $15,50 \mathrm{~m}$ en Baie du Mont Saint-Michel et 13,50 m à l'embouchure de La Rance.

La Rance est une rivière modeste, longue de $100 \mathrm{~km}$ environ, à faible débit $\left(7 \mathrm{~m}^{3} / \mathrm{sec}\right.$. en moyenne). L'estuaire qui sur le plan morphologique se présente comme une succession de goulets étroits et de larges bassins, s'étend sur une vingtaine de kilomètres.

C'est au débouché de ce petit fleuve entre les villes de Dinard et Saint-Malo que fut établie l'usine marémotrice. Débutée en 1963 sa construction, achevée en 1966, aboutit à la création d'un réservoir de 184 millions de $\mathrm{m}^{3}$ utiles occupant une surface de 2200 hectares à la cote $+13,50 \mathrm{~m}$ (fig. 1).

\section{L'ouvrage marémoteur}

D'une longueur de $750 \mathrm{~m}$ il est ancré sur des fonds granitiques à $13 \mathrm{~m}$ au-dessous du 0 des cartes marines. Supportant une route à grande circulation il comprend d'ouest en est, une écluse, l'usine proprement dite avec ses 24 groupes bulbes, une digue morte et enfin un barrage mobile large de $115 \mathrm{~m}$ formé de 6 vannes qui peuvent assurer le passage d'un débit total de $9600 \mathrm{~m}^{3} / \mathrm{sec}$. sous une dénivellation de 5 mètres (fig. 2).

Chaque groupe bulbe inclut, outre un alternateur de $10 \mathrm{MW}$, une turbine horizontale à 4 pales tournant à 94 tours/minute et capable d'absorber un débit de $275 \mathrm{~m}^{3} / \mathrm{sec}$. Grâce à ces bulbes qui autorisent le turbinage dans les deux sens d'écoulement de l'eau, l'énergie peut être produite tant au remplissage qu'au vidage du bassin : ce type de fonctionnement est qualifié de cycle à double effet (fig. 3).

A la lumière de cette description très sommaire de l'ouvrage il apparaît clairement que les échanges d'eau se réalisent à travers l'ensemble "turbines + vannes » de la mer vers le bassin et par l'intermédiaire des seules turbines en sens inverse.

\section{Les effets sur l'environnement}

De ce point de vue il convient de distinguer les conséquences de la construction de l'ouvrage de celles dues au fonctionnement de l'usine.

\subsection{Les effets de la construction}

La construction fut réalisée à sec à l'intérieur de trois enceintes de batardeaux. A l'exception de vidanges bihebdomadaires de faible amplitude $(1 \mathrm{~m})$, à caractère sanitaire, l'estuaire fut isolé de la mer ouverte durant trois années, de 1963 à 1966 (Rouvillois, 1967).

Pendant toute cette période le niveau du plan d'eau fut stabilisé à la cote $+8,50 \mathrm{~m}$. Il s'ensuivit d'importantes fluctuations de la salinité des eaux du bassin dont les valeurs s'abaissèrent jusqu'à $10 \%$ à proximité du barrage et un accroissement considérable du taux de sédimentation des particules fines. La transformation d'un estuaire à fort marnage en un bassin à niveau constant provoqua la 
disparition quasi totale de la flore et de la faune marines; en contrepartie quelques populations d'espèces d'invertébrés et de poissons très euryèces se développèrent abondamment.

\subsection{Les effets du fonctionnement sur le milieu}

Les contraintes d'exploitation de l'usine impriment aux eaux du bassin un régime "de marée " bien particulier. Il se démarque de celui observé en mer ouverte par la réduction du marnage qui oscille entre 4 et $5,50 \mathrm{~m}$ selon que les bulbes turbinent ou non dans les deux sens d'écoulement des eaux, par la remontée du niveau moyen $(2,50 \mathrm{~m}$ environ) et la durée d'immobilisation des masses d'eau spécialement longue aux niveaux hauts.

La réduction du marnage a pour corollaire celle de la superficie du domaine intertidal. Evaluée, avant l'aménagement et en marée de vive-eau, à $70 \%$ de la surface totale du bassin, la zone découvrante n'en représente plus aujourd'hui que $50 \%$ dans le cas d'un fonctionnement normal (simple effet en vive-eau et double effet en morte-eau).

Avant l'aménagement La Rance correspondait du point de vue hydrologique à une ria à stratification haline peu marquée le long de laquelle les auteurs reconnaissaient un secteur aval maritime suivi de deux sections, la première à salinité forte caractérisée par deux régimes saisonniers relayée par une seconde à salinité faible (FISCHER, 1929, 1931, 1933 ; MARIE, 1938 ; Priou, 1947). Aujourd'hui deux parties seulement peuvent être individualisées, le réservoir marin dont la salinité des eaux de fond reste supérieure à $30 \%$, et le véritable estuaire en amont (RÉTIÈRE 1979; RÉTIÈRE et RICHARD, 1980). La création d'un bassin aux variations de niveaux d'amplitude modeste a entraîné le recul et la réduction de la superficie de la zone estuarienne et atténué les fluctuations saisonnières de la salinité des eaux (fig. 4).

Les violents courants de vannage et de turbinage ont modifié la distribution des sédiments dont l'origine, la nature, la texture et la répartition avaient été bien étudiées entre 1954 et 1959 (BERTHOIS et BERTHOIS, 1954 et 1955; Bourcart et Roa Morales, 1957 ; Bourcart, 1959). En effet dès 1971 RÉTIÈrE constate qu'à proximité du barrage certains fonds ont été érodés, des bancs de sable déplacés et que des sédiments "ventilés" ont plus ou moins recouvert les fonds de cailloutis qui occupent le lit de La Rance dans sa partie avale (RÉTIÈRE, 1979). Alors que l'intensité de l'hydrodynamisme a surtout remanié la distribution des sédiments grossiers, les longues durées d'étale ont au contraire favorisé le dépôt des particules fines dans les anses et les baies (RÉTIÉRE et RICHARD, 1981). Cependant le bilan sédimentaire semble traduire une évolution naturelle de l'estuaire probablement liée à l'absence de réservoir important de matériel en suspension dans les eaux côtières près de l'embouchure de La Rance (HAN, 1982). Ces changements, d'ampleur somme toute limitée (L.C.H.F., 1982) se sont d'ailleurs plus nettement manifestés au cours des premières années qui ont suivi la mise en service de l'ouvrage (fig. 5).
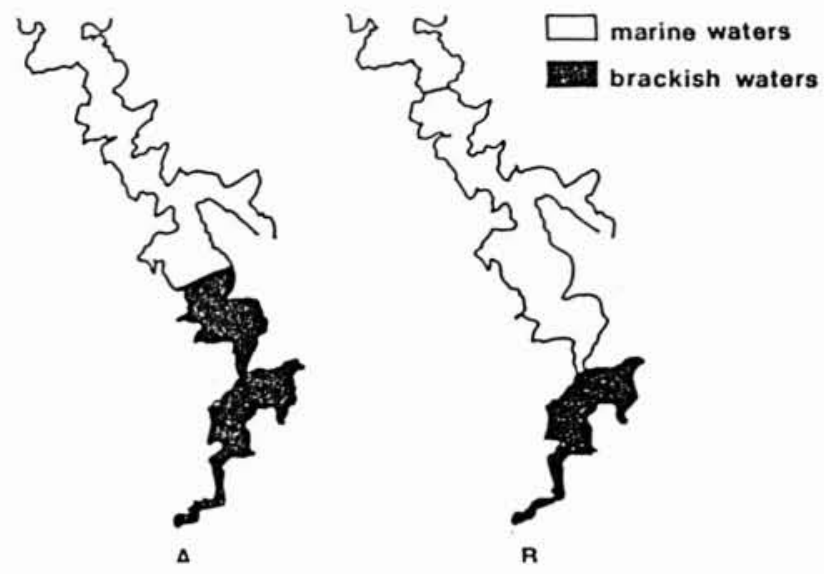

4. Extension des zones estuariennes avant $(A)$ et après (B) la construction du barrage. (D'après RÉTIĖRE et RICHARD, 1980). Marine waters : eaux marines.

Brackish waters : eaux estuariennes.

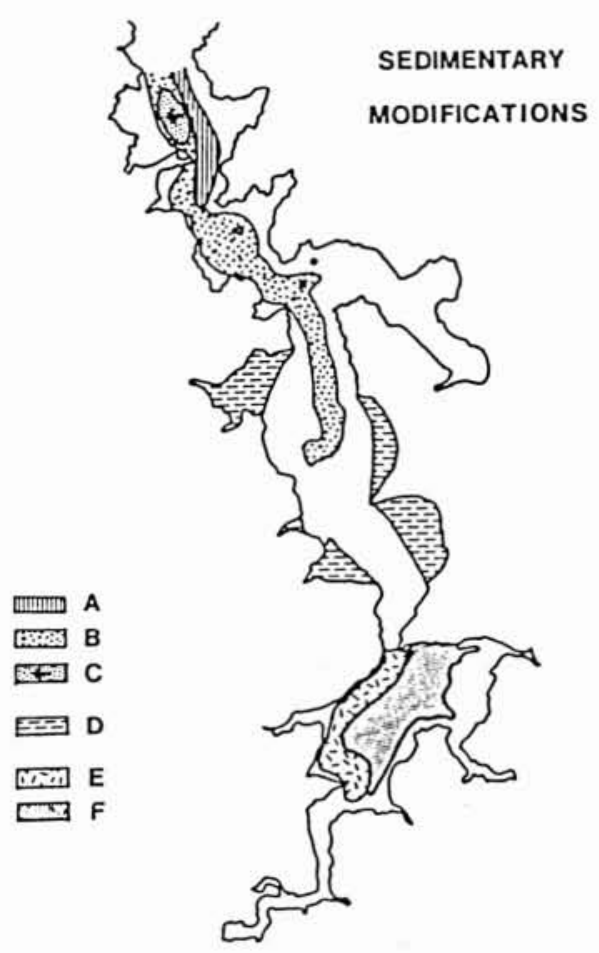

5. Modifications sédimentaires. (D'après RETIĖRE et RICHARD, 1980.)

A : fonds érodés. B : cailloutis localement ennoyés dans des sables grossiers. C : banc de sable déplacé. D : zones de dépôt de sédiments fins. $\mathrm{E}$ : sables propres hétérogènes. $\mathrm{F}$ : dépôts vaseux. 


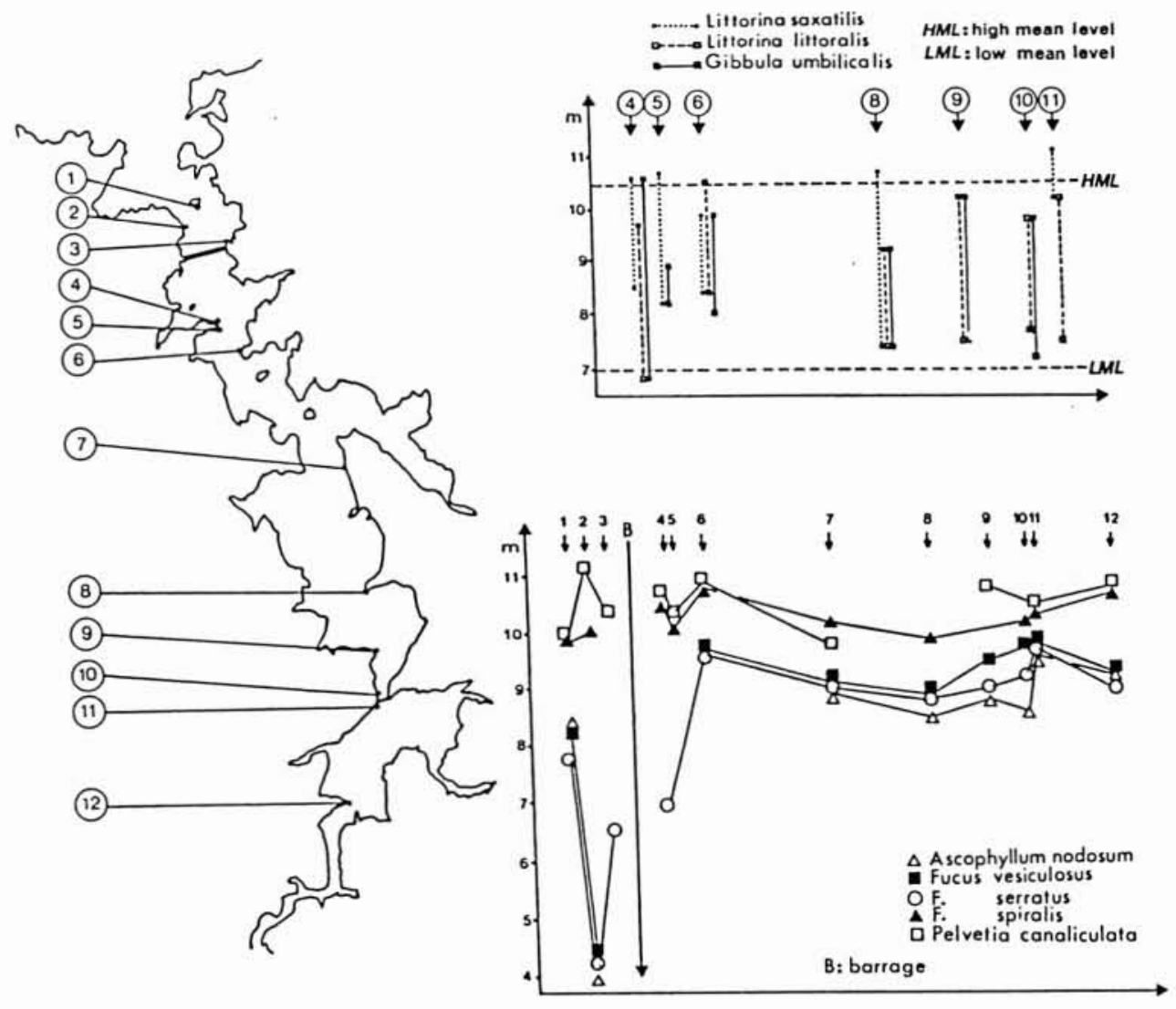

6. Distribution verticale de 5 espèces d'algues brunes (Ascophyllum nodosum. Fucus vesiculosus, Fucus serratus, Fucus spiralis et Pelvetia canaliculata) et de trois espèces de mollusques gastéropodes (Littorina saxatilis, Littorina littoralis, Gibbula umbilicalis) en aval et en amont du barrage jusqu'à l'écluse du Chatelier. (D'après LANG, 1986.)

\subsection{Les conséquences écologiques du fonctionnement}

\subsubsection{Distribution des espèces et des communautés}

En préalable à l'exposé des résultats concernant la distribution des espèces, la structure des communautés et les modalités de leur installation, il convient au travers des données relatives à certains groupes zoologiques inventoriés avec une particulière attention ou ayant fait l'objet de recherches à caractère écologique de souligner la richesse spécifique du bassin après 20 années de fonctionnement de l'usine. Ainsi né dénombre-t-on pas moins de 110 espèces d'annélides polychètes (RÉTIÉRE, 1979; LECHAPT, 1983 ; LANG, 1986), 47 espèces de crustacés décapodes - contre 44 avant l'implantation du barrage - (Le Calvez, 1986) et environ 70 espèces de poissons constituant des peuplements dont la composition et la diversité sont comparables à celles de leurs homologues de Manche occidentale (LE MAO, 1985). Nous illustrerons les types de répartition des espèces sur les deux exemples de la faune des substrats durs de la zone artificiellement découvrante qualifiée «d'inter niveaux " et de celle peuplant les fonds meubles non exondables.
Distribution des espèces des substrats durs de «l'inter niveaux $»$

Récemment LANG a montré (1986) que les végétaux qui marquent la zonation du domaine intertidal sont présents dans le bassin (Lichens, Fucales, Laminaires); toutefois leur extension verticale reste limitée (5 à 6 mètres au lieu de 13). Les algues qui occupent le long du littoral les niveaux bas de la zone de balancement des marées voient leur limite supérieure de répartition remonter considérablement dans le bassin. Il en est d'ailleurs de même pour les invertébrés (gastéropodes principalement) vivant normalement parmi ces algues. La succession des niveaux supérieurs atteints par l'ensemble des organismes étudiés pourrait laisser croire à leur étagement. Pourtant dès lors que l'on prend en compte l'amplitude totale de leur distribution verticale il n'est plus possible de reconnaître une réelle zonation (fig. 6). Cette apparente dualité trouve son explication dans l'analyse comparée des rythmes de variations du niveau du plan d'eau du bassin et des hautes et basses mers à l'extérieur de l'estuaire. De fait, dans le bassin les variations des niveaux hauts et bas, irrégulières, souvent hachées et susceptibles d'amples fluctuations d'un 


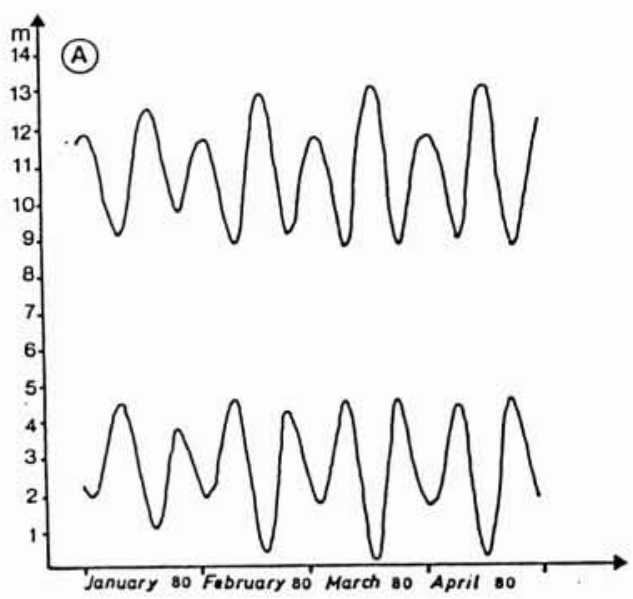

7. Courbes de marée (A) et de variations de niveaux hauts et bas dans le bassin (B) respectivement entre janvier-avril 1980 et février-juin 1981. (D'après LANG, 1986.)

8. Carte des peuplements benthiques.

A : Peuplement des sables grossiers à Spisula elleptica - Spisula ovalis - Saccocirrus papillocercus.

B : Peuplement des sables fins vaseux à Abra alba - Melinna palmata.

C: Faciès appauvri du peuplement à Abra alba - Melinna palmata.

D : Peuplement des fonds durs à épibiose sessile.

E : Peuplement des sables propres à Nephtys cirrosa.

(D'après RÉTIÈRE, 1979.)

Comparaison des cartes de distribution de Melinna palmata en 1971 et 1976 montrant la stabilité de la limite de pénétration de l'espèce dans la partie amont du bassin. (D'après RÉTIÉRE et RICHARD, 1980.)

9. Tendance évolutive du peuplement.

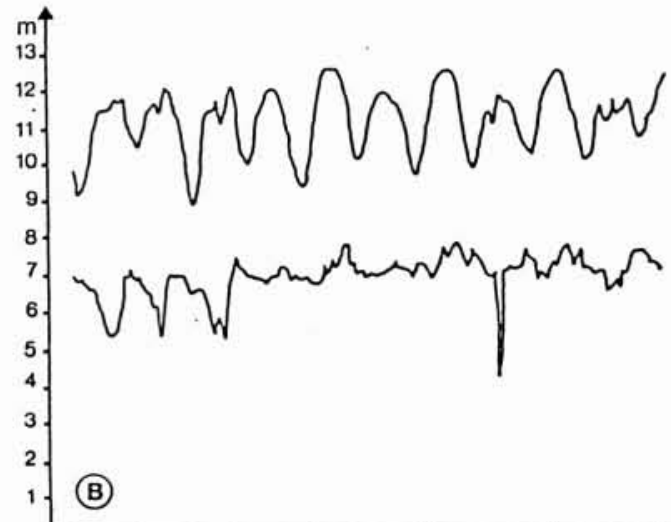

ISOLEMENT

"REOUVERTURE" DEBUT DE

DE L'ESTUAIRE L'ECHANTILLONAGe

$\frac{1}{1963}, \frac{1}{1963^{\prime}}, \frac{1}{1971^{\prime},},{ }^{1},{ }_{1982}^{\prime}$

STABILITE HYDROLOGIQUE $(\mathrm{S} \%$ )

\begin{tabular}{|c|c|}
\hline $\begin{array}{c}\text { Augmentation du nombre et } \\
\text { de la densité des espèces } \\
\text { principales }\end{array}$ & $\begin{array}{l}\text { Stabilisation du nom- } \\
\text { bre d'espèces princ1- } \\
\text { pales et évolution } \\
\text { 1ente de la dens1té }\end{array}$ \\
\hline Equilibration de la hiérarchie des espèces principales \\
\hline
\end{tabular}

EVOLUTION NATURELLE
ETABLISSEMENT DU PEUPLEMENT
SOUS LE CONTROLE DE LA DU PEUPLEMENT CONTROLEE
PERTURBATION INITIALE

7.

Fobruary 81 Mareh 81 April o1 May 81 June s1

SOUS LE CONTROLE DE

JEU DES RELATIONS INTER ET INTRA-SPECIFIQUES

8.

Benthic communities in the maritim basin of the RANCE 1971

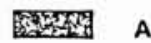

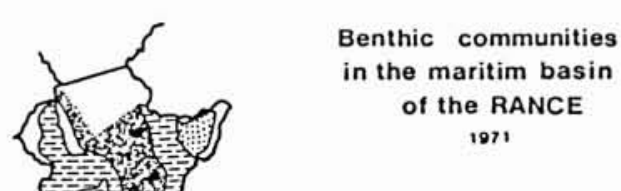

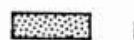

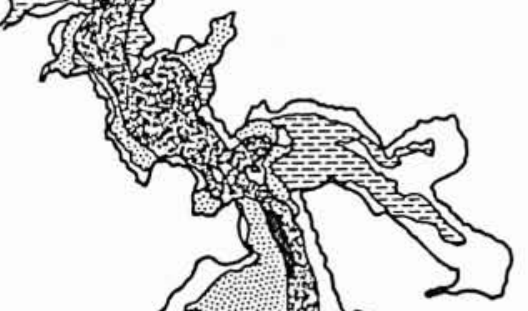

?

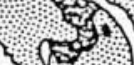

医포고

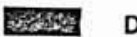

F⿸尸 $E$

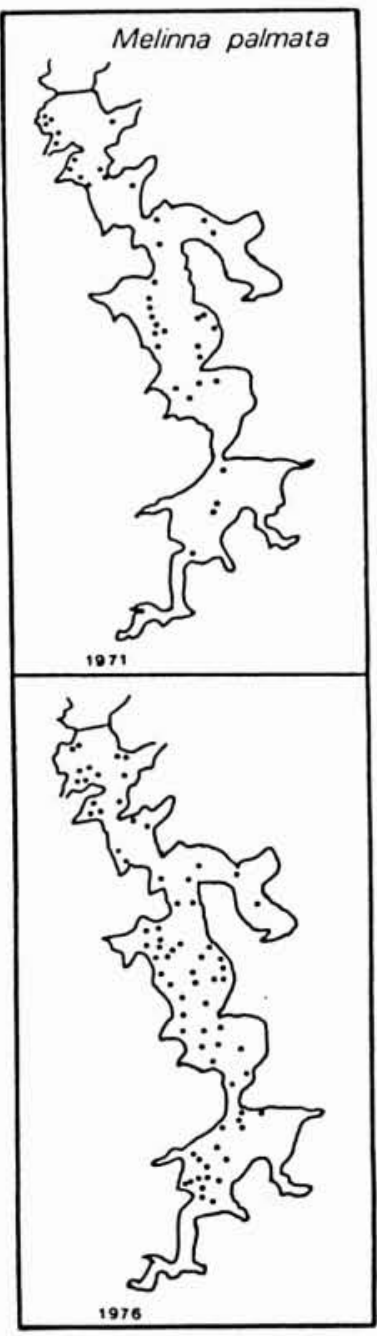


jour à l'autre ne sont pas toujours synchrones. En outre la duree des niveaux hauts peut être longue et atteindre 8 heures par cycle de fonctionnement (fig. 7). Il résulte de cette instabilité des conditions d'émersion-immersion, l'installation dans l'interniveau d'un peuplement unique au sein duquel ne se manifeste pas clairement la zonation des ceintures décrite en domaine intertidal.

Les espèces et les communautés des fonds non exondables

Dès 1971 RÉTIĖRE a identifié en Rance quatre communautés benthiques à savoir : le peuplement des sédiments fins plus ou moins envasés à Abra alba - Corbula gibba, ceux des sables fins à moyens propres à Nephtys cirrosa et des sables grossiers à Spisula ovalis - Spisula elliptica Saccocirus papillocarcus et enfin celui des cailloutis et fonds durs à épibiose sessile (fig. 8).

Cinq années ont donc été un délai suffisant pour que s'établisse sous une forme, certes encore peu diversifiée, et sur certains fonds peuplés auparavant par une faune intertidale, des communautés subtidales présentes à la périphérie du golfe normand-breton (RÉTIẺRE, 1975 et 1979).

La répartition longitudinale des espèces appartenant aux peuplements estuariens ou à leurs faciès est sous la dépendance conjointe des facteurs édaphiques et hydrologiques. De ce point de vue il est intéressant de remarquer que le recul du secteur à salinité variable a coïncidé avec celui de la zone de raréfaction des espèces marines (FISCHER, 1929 et 1931 ; RÉTIÈrE et RiCHARD, 1981).

Enfin, de la comparaison de cartes de distribution d'espèces établies à cinq années d'intervalle (1971-1976) il ressort nettement qu'à la stabilité des facteurs abiotiques répond celle de la répartition spatiale des organismes (RÉTIĖRE et RICHARD, 1981 ; LECHAPT, 1983).

Des études floro-faunistiques et écologiques à caractère exclusivement descriptif se dégagent des enseignements majeurs :

- la flore et la faune se sont réintroduites dans le bassin après qu'il ait été défauné pendant la phase d'isolement de l'estuaire ;

- la faune est actuellement très diversifiée;

- les schémas de distribution longitudinale et verticale des espèces illustrent l'ajustement biologique aux nouvelles conditions de l'environnement.

L'ouvrage marémoteur se caractérise donc par sa perméabilité biologique qui a permis la recolonisation du bassin à partir de la mer ouverte.

\subsubsection{Les modalités de recolonisation du bassin}

De 1972 à 1982 le suivi des principales composantes endogées du faciès à Melinna palmata du peuplement à $\mathrm{Abra}$ alba - Corbula gibba révèle qu'à une phase d'accroissement de la richesse spécifique et de la densité (s'étendant jusqu'en 1976) succède une période de stabilisation (CLAVIER et al., 1982). Or, pendant toute la durée d'observation, six populations d'annélides polychètes représentent à elles seules $80 \%$ de l'abondance de l'endofaune et $90 \%$ de sa biomasse contrôlant ainsi largement la dynamique du peuplement. Leur étude (RÉTIÈre, 1976 et 1979 ; Clavier, 1981 ; RIVAIN, 1983 ; LECHAPT, 1983) a bien fait apparaître que la recolonisation s'est réalisée aux dépens des populations les plus proches de l'estuaire et susceptibles d'occuper le biotope, indépendamment de la durée de vie des espèces et de la longueur de la phase pélagique de leur cycle ; le transport passif d'adultes a pu également contribuer de manière non négligeable à leur installation dans le bassin de La Rance.

Malgré une stabilisation rapide des conditions environnementales après la mise en service de l'ouvrage, dix années ont donc été nécessaires à l'établissement d'un nouvel équilibre du peuplement (1966-1976) qui a ensuite évolué par le jeu naturel des relations intra et interspécifiques sans relation avec la perturbation initiale, les espèces à durée de vie longue imprimant la tendance générale (fig. 9).

\subsubsection{La biologie des espèces}

De l'ensemble des connaissances acquises sur la biologie des organismes nous extraierons, à titre d'exemple celles ayant trait à la reproduction et la croissance d'un invertébré, le mollusque gastéropode Haliotis tuberculata et des poissons peuplant ou fréquentant les eaux du bassin.

L'ormeau Haliotis tuberculata est présent en forte densité sur les côtes nord de Bretagne (GAILLARD, 1958). Selon ClAVIER et Richard (1985) il habite, dans la région de Saint-Malo, les anfractuosités sur fonds durs ou de forte granulométrie. Ces auteurs ont été conduits, dans le cadre de leurs recherches, à comparer des populations vivant sur le littoral et dans le bassin de La Rance : il s'avère que si dans ce dernier site la période de ponte est légèrement plus étalée, la croissance n'y est pas significativement différente.

L'étude des stades de maturité des gonades couplée à celle de l'ichtyoplancton et à l'observation des pontes fixées conduit LE MAO (1985) à affirmer que sur les 70 espèces de poissons qui peuplent les divers fonds de La Rance ou en occupent le domaine pélagique pas moins de 30 s'y reproduisent de façon certaine. Profondément transformée La Rance continue donc de jouer un rôle essentiel dans le cycle de vie de nombreuses espèces servant de frayères pour certaines (Clupea harengus) et (ou) de nurseries pour d'autres (Pollachius pollachius). Beaucoup de petits poissons littoraux y réalisent la totalité de leur cycle bien que leurs populations puissent bénéficier d'un fort recrutement en larves ou juvéniles venant de la mer ouverte (Ciliata mustela, Spinachia spinachia) ou voir leurs effectifs reproducteurs s'accroître par l'arrivée de géniteurs (Atherina presbyter). Enfin La Rance assure envers les espèces catadromes (Anguilla anguilla, Conger conger) la fonction de tout estuaire laissant pénétrer les stades larvaires et ressortir les géniteurs. En règle générale la croissance linéaire des poissons étudiés par LE MAO (1985) ne semble guère perturbée. Les bars Dicentrarchus labrax ont une croissance, certes lente, mais très comparable à celle observée en Irlande et au Pays-de-Galles c'est-à-dire en limite nord de l'aire d'abondance de cette espèce méditerranéolusitanienne. Il en est de même de la croissance de la daurade, Liza aurata qui reste proche de celle signalée en d'autres secteurs de Bretagne. Pour le hareng, Clupea 
Tableau 1

\begin{tabular}{|l|c|c|}
\hline & La Rance & Rivière de Morlaix \\
\hline Vasières exondables & $8,5(1)$ & $9,5(3)$ \\
\hline Sables vaseux non exondables & $15,0-18,0(2)$ & \\
\hline
\end{tabular}

Biomasse moyenne annuelle $\left(\mathrm{g} / \mathrm{m}^{2}\right)$ des espèces d'endofaune des communautés des sables vaseux dans deux estuaires bretons : La Rance et La Rivière de Morlaix.

(1) 1979-1980: Clavier, 1981.

(2) 1972-1973: RÉTIËRE, 1979 (en deux sites différents)

(3) 1977-1978: DAUVIN, 1984.

Tableau 2

\begin{tabular}{|c|c|c|c|c|c|}
\hline & & \multicolumn{2}{|c|}{ Vasières exondables } & \multicolumn{2}{|c|}{ Sables vaseux non exondables } \\
\hline & & $P$ & $P / B$ & & $P / B$ \\
\hline La Rance & $\stackrel{\mathrm{A}}{\mathrm{N}}$ & $1,5-9,0$ & & \multirow{2}{*}{6,0} & \multirow{2}{*}{$1,2(4)$} \\
\hline River Lyhner & $\begin{array}{l}\mathrm{A} \\
\mathrm{N}\end{array}$ & $\begin{array}{l}0,1-12,5 \\
6,0-2,8\end{array}$ & $\begin{array}{l}4,0-5,2(2) \\
1,4-0,8(3)\end{array}$ & & \\
\hline
\end{tabular}

Production exprimée en $\mathrm{g} / \mathrm{m}^{2} /$ an de Ampharete acutitrons (A) et Nephtys hombergii (N) dans deux estuaires: La Rance (France) et la River Lyhner (Grande-Bretagne).

(1) 1979-1980: ClaVier, 1981 (en deux sites différents).

(2) et (3) 1972-1977: WARWICK and PRICE (1980).

(4) 1972-1973: RÉTIÈRE (1979).

harengus le bassin de La Rance se révèle même un milieu particulièrement favorable. L'interprétation des résultats relatifs à la croissance pondérale est rendue délicate par le très petit nombre d'espèce prises en compte et l'insuffisance de données de références : alors que la croissance du bar Dicentrachus labrax est voisine en Rance et sur la côte Atlantique française celle de la daurade Liza aurata est moins bonne. Ces enseignements auxquels il convient d'ajouter ceux concernant d'autres invertébrés, en particulier des annélides polychètes, convergent pour laisser penser que la reproduction et la croissance des organismes ne sont pas affectées par les nouvelles conditions écologiques du bassin de La Rance.

\subsubsection{Le fonctionnement de l'écosystème}

\subsubsection{La production primaire}

Dans le contexte de leurs recherches sur la réalisation de pollutions expérimentales à grande échelle dans le bassin de La Rance, LACAZE et al. (1972) ont mis en évidence une augmentation importante de la production primaire d'aval en amont. Ce gradient peu marqué en hiver s'affirme nettement au printemps, les eaux de surface demeurant toujours les plus productives. Les valeurs correspondant au secteur marin sont supérieures à celles des eaux de la baie jouxtant l'estuaire et deux à quatre fois plus fortes que celles observées plus à l'ouest, en mer ouverte, sur le littoral breton, dans les parages de Roscoff (GraLL, 1972). Les mouvements d'eau sont probablement responsables du maintien à un niveau élevé de la productivité du bassin sans qu'apparaissent d'eutrophisations excessives ou de variations soudaines de la production primaire.

\subsubsection{Biomasse et production macrozoobenthique}

Les biomasses et production sont susceptibles de fluctuer d'une année à l'autre. Cette réserve exprimée, il n'en demeure pas moins que les valeurs de biomasse moyenne annuelle relatives au peuplement à Abra alba - Corbula gibba des sables vaseux non exondables de La Rance (RÉTIÈRE, 1979) sont approximativement doubles de celles se rapportant d'une part à la faune endogée des vasières découvrantes (CLAVIER, 1981) et d'autre part à la communauté subtidale établie dans des sédiments de texture très voisine à l'embouchure de la rivière de Morlaix, près de Roscoff, sur la côte armoricaine (tabl. I). L'amplitude des variations, selon les sites et les années, des valeurs de production et des rapports $P / B$ de deux populations annélidiennes, Nephtys hombergii et Ampharete acutifrons étudiées en Rance (RÉTIÈrE, 1979 ; ClAVIER, 1981) et dans la rivière Lyhner en Grande-Bretagne (WARWICK et PRICE, 1980) est également du même ordre de grandeur (tabl. 2). 

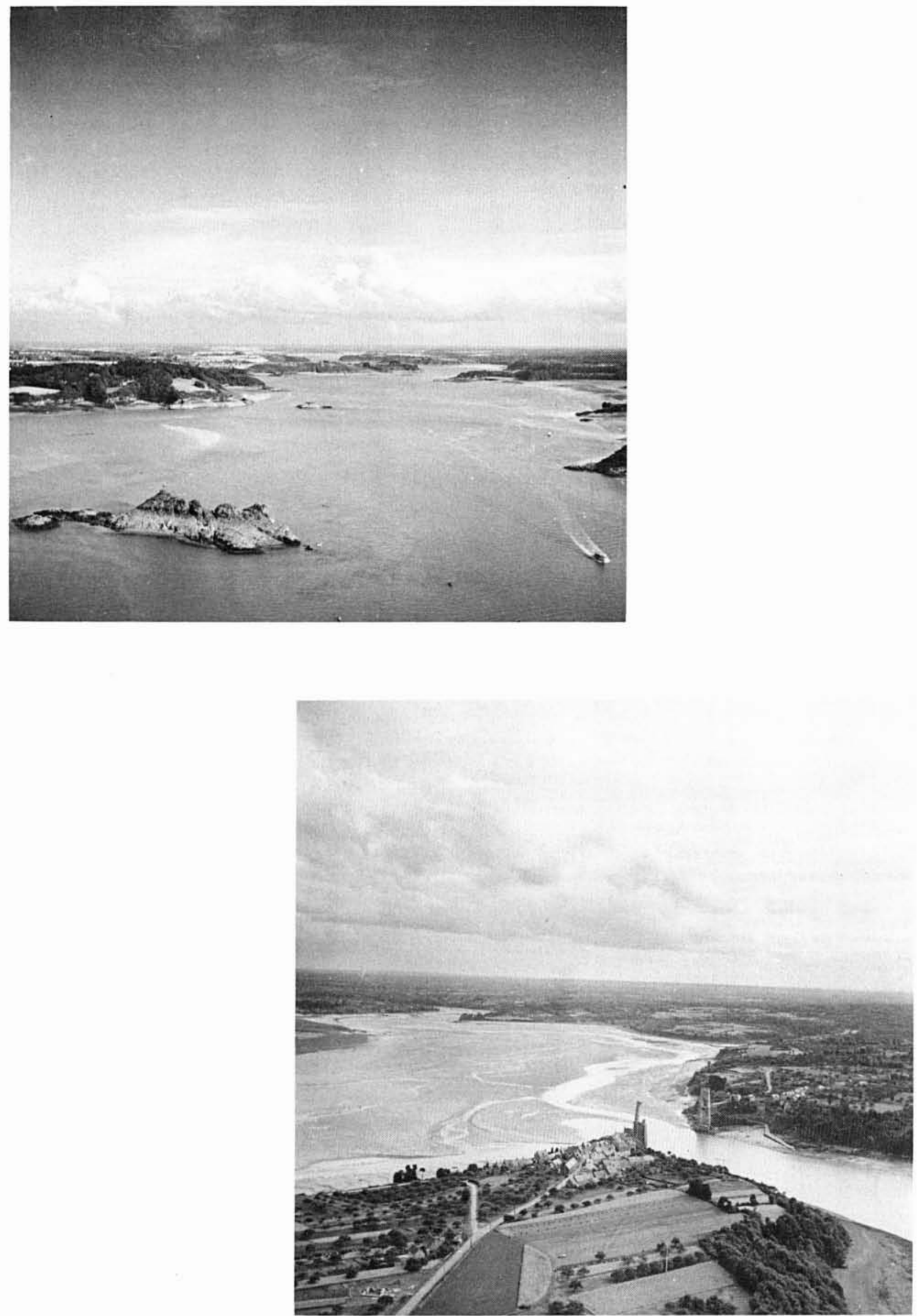

LA HOUILLE BLANCHE $/ \mathrm{N}^{\circ} 2-1989$ 


\subsubsection{Déplacements et migrations}

En règle générale les estuaires, zones de transition entre les eaux côtières et dulçaquicoles, sont des voies de passage essentielles pour de nombreuses espèces; les mouvements qui les animent sont d'amplitude variée et affectent une ou plusieurs phases de leur cycle de vie. Une question primordiale se pose donc : la présence du barrage et le fonctionnement des turbines entravent-ils leurs déplacements? Ce problème a été finement analysé par LE MAO (1984 et 1985) qui s'est intéressé, outre à la faune piscicole, au cas de deux invertébrés d'intérêt économique, l'un marcheur, l'araignée de mer Maia squinado, l'autre nageur, la seiche, Sepia officinalis.

\section{L'araignée de mer Maia squinado}

Parmi les espèces de crustacés d'intérêt halieutique, l'araignée de mer est sans conteste la plus abondante. Alors que les immatures occupent le bassin tout au long de l'année en densité cependant plus faible en hiver, les adultes manifestent un vrai comportement migrateur (DE KERGARIOU, 1971). Au printemps, leur arrivée régulière qui donne d'ailleurs lieu à exploitation pendant toute la période de présence des individus reproducteurs dans le bassin est la preuve que le barrage ne constitue pas pour ce migrateur « marcheur" un véritable obstacle.

\section{La seiche Sepia officinalis}

La seiche est un mollusque céphalopode dont les habitudes migratoires sont fort complexes. Dans la baie de SaintMalo les adultes apparaissent au début du mois d'avril suivis des immatures qui deviennent abondants à partir de juin. Le départ de la majorité de ces seiches se produit au cours du mois d'octobre, quelques individus demeurant à la côte jusqu'à la fin novembre. Les juvéniles issus de la ponte estivale échelonnent leur départ d'octobre à fin novembre. Bien que leurs zones d'hivernage soient encore imprécises il est raisonnable, compte tenu des caractéristiques thermiques des eaux, de les situer à l'ouest de La Manche et en Mer Celtique (fig. 10).

Malgré son aspect saisonnier la pêche de la seiche en
Rance est une activité halieutique primordiale. En 1982, d'avril à juin, LE MAO (1985) estime la production entre 25 et 35 tonnes, celle-ci pouvant même atteindre, les bonnes années et de part et d'autre du barrage, 40 tonnes. Le succès de la reproduction dans le bassin joint à la pêche intensive qui s'y exerce et les fortes quantités capturées témoignent de la perméabilité de l'ouvrage marémoteur à ces céphalopodes de grande taille.

La faune piscicole

La présence en Rance d'espèces à cycle complexe exigeant un séjour au large implique nécessairement des migrations et des mouvements erratiques entre le bassin et la mer ouverte: en ce domaine il convient de faire la part des vannes et des turbines dans ces échanges. Pour tenter de répondre à cette question LE MAO (1985) a prélevé mensuellement de septembre 1983 à août 1984 des échantillons de part et d'autre des groupes bulbes, dans les perthuis de batardeaux : quarante-quatre espèces ont ainsi été récoltées. Elles appartiennent à trois catégories: les formes pélagiques dominent largement le groupe des poissons bentho-démersaux et celui des espèces associées aux algues en épave. Mais il est à noter que dans tous les cas les maxima d'abondance dans les perthuis coïncident avec les périodes de reproduction ou de concentration trophique au voisinage du barrage: le passage par les turbines est donc effectif et se produit à la faveur des mouvements migratoires.

De l'étude des blessures des poissons et céphalopodes pêchés dans les perthuis et capturés de part et d'autre du barrage LE MAO conclut que le passage de ces organismes au travers des turbines semble se réaliser sans problèmes majeurs en raison des caractéristiques des groupes bulbes (grand diamètre et faible vitesse de rotation). Une mortalité indirecte sans doute négligeable au regard du nombre considérable de poissons transitant par les turbines est cependant décelable. La traversée de ces structures désorganise en effet les bancs favorisant alors la prédation par les oiseaux piscivores. (Laridés mais également cormorans Phalacrocorax carbo et P. aristotelis et pingoins Alca torda.)

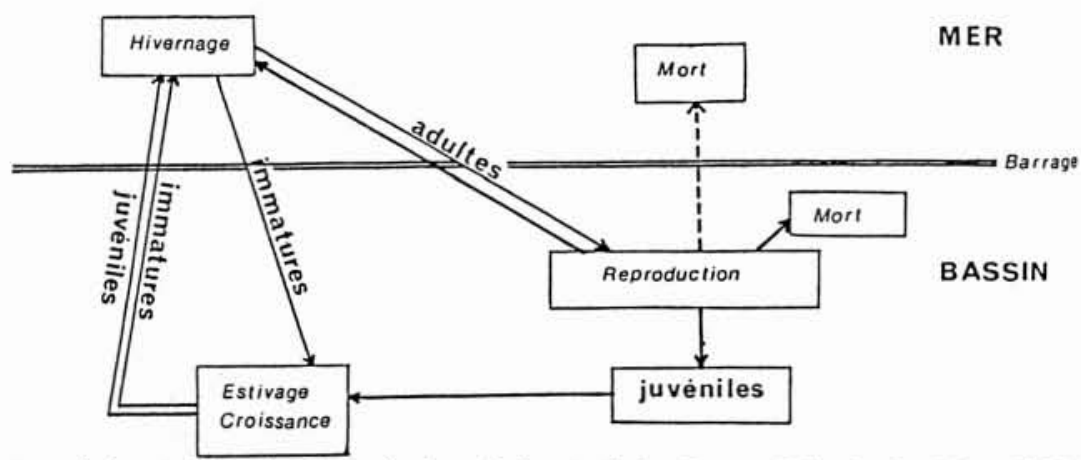

10. Cycle biologique de la seiche Sepia officinalis dans le bassin de La Rance. (D'après LE MAO, 1985.) 


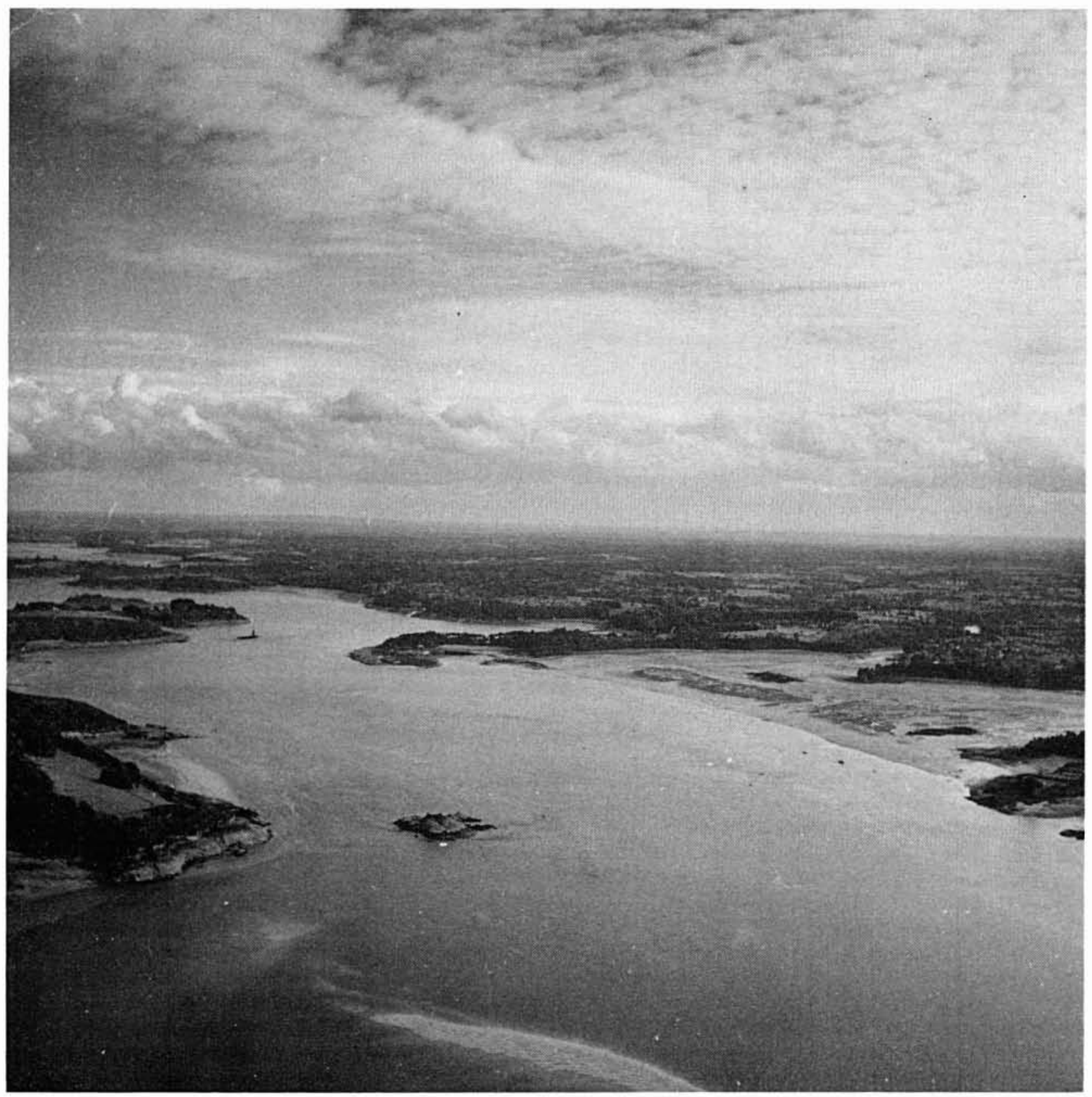


Tableau 3

\begin{tabular}{|c|c|c|c|c|c|}
\hline & \multicolumn{5}{|c|}{ Cerastoderma sp. juv. } \\
\hline & avril & mai & juin & juillet & août \\
\hline $\begin{array}{l}\text { Solea vulgaris } \\
\text { Pleuronectes platessa } \\
\text { Pomatoschistus minutus } \\
\text { Callionymus lyra }\end{array}$ & $\begin{array}{l}\cdots \\
0 \\
0 \\
\cdots\end{array}$ & $\begin{array}{l}\cdots \\
0 \\
0\end{array}$ & $\begin{array}{r}105 \\
2 \\
2 \\
0\end{array}$ & $\begin{array}{c}191^{*} \\
109^{*} \\
2 \\
6\end{array}$ & $\begin{array}{r}0 \\
628 \\
0 \\
2\end{array}$ \\
\hline $\begin{array}{l}\text { Solea vulgaris } \\
\text { Pleuronectes platessa } \\
\text { Pomatoschistus minutus } \\
\text { Callionymus lyra }\end{array}$ & $\begin{array}{c}33^{*} \\
155^{*} \\
\ldots-\end{array}$ & $\begin{array}{l}121^{*} \\
138^{*} \\
\ldots\end{array}$ & $\begin{array}{r}\text { rete ac } \\
0^{*} \\
71^{*} \\
34^{*} \\
105^{*}\end{array}$ & $\begin{array}{c}1 \\
104^{*} \\
13 \\
488^{*}\end{array}$ & $\begin{array}{c}62^{*} \\
53 \\
254^{*} \\
17\end{array}$ \\
\hline
\end{tabular}

Les astérisques soulignent les cas réels de compétition trophiques selon les critères définis par GodFriAUX et QuiNiOU.

Valeur mensuelle de l'index de RiCHARD calculé pour les poissons prédateurs dominants et les deux espèces principales de proies Ampharete acutifrons et juvéniles de Cerastoderma sp. (d'après LE MAO, 1986).

\subsubsection{Relations trophiques}

Nature des relations trophiques

Après avoir défini les peuplements de poissons et céphalopodes du bassin de La Rance LE MAO (1985) a mis en évidence les relations d'ordre alimentaire qu'ils entretiennent avec les autres composantes floro-faunistiques de l'écosystème.

Au sein du peuplement pélagique cet auteur distingue deux catégories principales d'organismes:

- les planctonophages dont l'alimentation est dominée soit par les crustacés copépodes et les larves de décapodes (Sprattus sprattus, Sardina pilchardus, Atherina presbyter) soit par l'ichthyoplancton (Clupea harengus);

- les prédateurs pourchassant activement les poissons planctonophages ; il s'agit principalement des céphalopodes Loligo vulgaris, Loligo forbesi et des poissons Scomber scombrus et Trachurus trachurus. Quand des relations se développent entre des représentants pélagiques et benthiques elles se réalisent généralement au profit des seconds.

La structure trophique des peuplements des substrats durs inclut outre des poissons phytophages (Spondyliosoma cantharus juvénile, Blennius gattorugine...). des espèces s'attaquant au zoobenthos sessile (Blennius pholis...) ou vagile (Ciliata mustela, Trisopterus luscus, Symphodus melops...) et des espèces piscivores consommant aussi des crustacés décapodes (Pollachius pollachius, Dicentrarchus labrax, Enophrys bubalis).

Sur les fonds meubles quelques espèces se nourrissent préférentiellement aux dépens du méiobenthos permanent (Gobius niger) ou temporaire (juvéniles de Solea vulgaris et Pleuronectes platessa), d'autres telles la plie Pleuronectes platessa adulte ou Callionymus lyra consommant plutôt le macrobenthos endogé. Toutefois les crustacés décapodes vagiles constituent des proies intervenant pour une part non négligeable dans le régime alimentaire du bar Dicentrarchus labrax, de l'anguille Anguilla anguilla ou du flet Platichthys flesus... La seiche Sepia officinalis, prédateur puissant, occupe le sommet de la pyramide alimentaire du peuplement des fonds meubles apparemment plus hiérarchisée en secteur maritime qu'estuarien où crustacés et poissons benthiques occupent le même niveau trophique.

En conclusion trois enseignements majeurs se dégagent de cette étude :

- les chaînes alimentaires pélagiques sont typiques de zones côtières en l'absence de prédateurs océaniques de haut niveau (sélacien, grands scombridés, cétacés) ;

- la nature des relations trophiques entre poissons et céphalopodes d'une part et la faune des sédiments meubles d'autre part est conforme au schéma opposant classiquement, de ce point de vue, le domaine côtier marin et les zones estuariennes ;

- enfin la mise en place de chaînes alimentaires complexes semble assurer une exploitation satisfaisante par les poissons et céphalopodes des ressources trophiques du bassin de La Rance.

Fragilité des relations trophiques

Il importe de savoir si les liens tissés entre les différents compartiments écologiques du bassin de La Rance peuvent être affectés par les modalités d'exploitation de l'usine marémotrice. Or, il est admis que l'interprétation des mécanismes fonctionnels de tout système biologique est d'autant plus aisée que celui-ci est simple, c'est-à-dire que le nombre de ses composantes est faible. Dans cet esprit LE MAO (1986) a analysé les relations entre la faune piscicole et celle des sédiments meubles du secteur estuarien, zone de nurserie pour de nombreuses espèces de 
poissons pélagiques et bentho-démersaux qui ne constituent pas une communauté bien établie sa composition variant au rythme des périodes de recrutement et de migration. De fait, seules les espèces Pomatoschistus minutus, Pleuronectes platessa et Anguilla anguilla en sont les hôtes permanents; encore convient-il de remarquer que les densités des deux dernières espèces chutent fortement au cours des mois d'hiver. Dès lors $90 \%$ des espèces benthiques capturées sont représentées par la plie Pleuronectes platessa, la sole Solea vulgaris, le gobie Pomatoschistus minutus et Callyonimus lyra.

La compétition trophique qui existe entre les différentes espèces de poissons benthiques s'alimentant sur les communautés estuariennes oligospécifiques est généralement très intense. En Rance LE MAO a montré qu'une réelle compétition se manifeste entre la plie et la sole envers deux espèces d'invertébrés, l'annélide polychète Ampharete acutifrons et le mollusque bivalve Cerastoderma sp. En effet les juvéniles de Cerastoderma sp. sont intensément exploités par les jeunes soles dès le mois de juin ; en juillet les plies du groupe $\mathrm{O}$ entrent en active compétition pour cette ressource et en août elles en demeurent les seuls consommateurs. Ampharete acutifrons qui devient alors une proie majeure dans l'alimentation des soles tout en restant l'enjeu d'une compétition intense entre les autres prédateurs d'avril à août apparaît donc comme une ressource primordiale pour les poissons benthiques ( $t a b l .3$ ). Mais en Rance les deux espèces Cerastoderma sp. et Ampharete acutifrons ont une durée de vie brève. L'équilibre trophique est donc fragile et dépend du succès de leur recrutement que des variations brutales des conditions abiotiques, elles-mêmes fonction du rythme de fonctionnement de l'usine marémotrice, peuvent profondément affecter.

A cet égard les conséquences des variations soudaines de niveaux enregistrées en juin 1983 lors du passage d'un fonctionnement "simple effet" à un fonctionnement «double effet » se traduisant par l'exondation périodique de vasières constamment immergées sont très révélatrices. L'abondance des populations de jeunes plies Pleuronectes platessa a chuté d'environ $80 \%$ à une époque capitale puisque le recrutement de l'espèce s'arrête au mois de juillet. Cette diminution de la densité suivie rapidement d'une recolonisation par des gobies Pomatoschistus minutus, est due à un taux élevé de mortalité : de nombreux individus se retrouvèrent à sec sur la vasière ou soumis à une élévation de la température de l'eau des flaques dans lesquelles ils s'étaient réfugiés, devenant dans tous les cas, des proies faciles pour les oiseaux. LE MAO note également un accroissement du coefficient de vacuité des individus survivants qui ne peut être associé à une raréfaction des proies puisque, dès le mois suivant, la fréquence du ver Ampharete acutifrons augmente dans les contenus digestifs des gobies analysés.

La déstabilisation, même brève, des conditions environnementales par un changement du rythme d'exploitation de l'usine est donc susceptible de perturber durablement le fonctionnement de l'écosystème.

\subsubsection{Utilisation du bassin par les oiseaux aquatiques hivernants}

Chaque année La Rance accueille plusieurs milliers d'oiseaux hivernants.

Les recensements effectués de novembre 1984 à mars 1985 par LE MAO et al. (1986) en complément des observations à caractère plus ponctuel de SCHRICKE et BourGAULT (1982) et de celles qu'ils réalisèrent entre 1977 et 1984 révèlent tout d'abord que l'augmentation de la surface du plan d'eau permanent a été favorable aux palmipèdes plongeurs : ainsi de fortes concentrations de grèbes, en particulier Podiceps nigricollis, ont lieu au début de l'hiver dans la ria.

Il apparaît également que les densités de petits échassiers sont élevées en comparaison de celles calculées pour des zones côtières françaises ou anglaises. Tout se passe comme si la production des peuplements des vasières découvrantes aux dépens desquels s'alimentent ces populations aviennes, compensait la réduction des surfaces exondables.

Enfin l'accroissement progressif, dès 1970, des effectifs de nombreuses espèces hivernantes (Tadorna tadorna, Branta bernicla...), également enregistré en Baie du Mt St-Michel, traduit les fortes potentialités d'accueil de la ria.

L'aménagement marémoteur de l'estuaire n'a donc pas eu de conséquences néfastes sur l'avifaune.

\section{Conclusion}

Pendant toute la durée de construction de l'ouvrage marémoteur l'estuaire à fort marnage a été transformé en un bassin à niveau constant ; les conditions hydrologiques et sédimentaires en ont été profondément bouleversées. La résultante biologique s'est manifestée par une disparition quasi totale de la flore et de la faune marine et seules les espèces les plus tolérantes aux fluctuations des facteurs abiotiques se sont développées. Cette étape se révèle donc extrêmement dommageable pour l'environnement aquatique. Le rythme des variations de niveau du plan d'eau, déterminé par le mode d'exploitation de l'usine, a entraîné une diminution de la superficie du domaine intertidal, créé de nouvelles conditions hydrodynamiques et hydrologiques et abouti en quelques années à une redistribution des sédiments meubles du bassin.

Progressivement une flore et une faune marine s'y sont établies puis diversifiées; les modes de distribution et de regroupement des espèces en unités cénotiques reflètent, avec des nuances, un ajustement biologique aux nouvelles conditions environnementales. La colonisation s'est réalisée, tout d'abord aux dépens des populations les plus proches susceptibles d'occuper les différents biotopes par 
l'intermédiaire des stades larvaires de dissémination mais aussi de juvéniles et d'adultes entraînés passivement par les violents courants de vannage.

La reproduction et la croissance des invertébrés et vertébrés étudiés semblent se dérouler identiquement dans le réservoir et en mer ouverte. Les niveaux élevés de productions primaire et macrozoobenthique, la structure complexe du réseau trophique, la libre circulation, par passage à travers les vannes et les turbines des organismes erratiques ou migrateurs témoignent de relations fonctionnelles normales au sein du nouvel écosystème et entre celui-ci et les eaux côtières.

Les effets écologiques de 20 années de fonctionnement de l'usine marémotrice de La Rance sont donc loin d'être négatifs. Mais ils ne sont pas directement transposables à d'autres sites dans le monde où ils seront évidemment dépendants des caractéristiques propres à chacun d'eux.

Ainsi à titre d'exemple, le taux de sédimentation dépendra de la charge des eaux en particules en suspension et du mode d'exploitation de l'usine ; de même taille et fragilité des organismes d'une part et vitesse de rotation des turbines d'autre part interviendront dans la détermination du taux de mortalité lié au franchissement de l'ouvrage.
Pourtant des enseignements d'une portée scientifique générale se dégagent de l'ensemble des recherches poursuivies sur le bassin maritime de La Rance :

- un aménagement marémoteur est, à des degrés divers, biologiquement perméable: il autorise la recolonisation d'un milieu modifié et les échanges entre estuaire et eaux côtières ;

- un nouvel équilibre est atteint au terme d'une dizaine d'années ; le système fonctionne alors par le jeu des relations intra et interspécifiques indépendamment de la perturbation initiale ;

- ce nouvel équilibre est totalement dépendant du mode d'exploitation de l'usine : un rythme régulier stabilise les conditions abiotiques;

- des variations brutales des facteurs environnementaux (pour des raisons de production d'électricité ou d'entretien des ouvrages), même brèves, peuvent avoir des effets écologiques sévères perceptibles à différentes échelles d'espace et de temps (moyen et long terme).

En conclusion générale le succès d'un aménagement marémoteur repose sur la bonne adéquation, possible à définir, entre les contraintes d'exploitation et le respect des équilibres biologiques.

\section{Références}

Berthois L. et Berthois C., 1954. - Etude de la sédimentation dans l'estuaire de La Rance. I. Granulométrie des sédiments. Bull. Lab. Mar. Dinard, 40: 4-15.

Berthois L. et Berthois C., 1955. - Etude de la sédimentation dans l'estuaire de La Rance. II. Minéralogie des sédiments. Bull. Lab. Mar. Dinard, 41 : 3-18.

Bourcart J., 1959. - Carte de La Rance in cours de Pétrographie sédimentaire: les roches sédimentaires et leur genèse. C.D.U. Paris : $402 \mathrm{p}$.

Bourcart J. et Roa Morales P., 1957. - Les dépôts vaseux de La Rance maritime et du Mont Saint-Michel. Bull. Soc. Géol. Fr., VII (4-5) : 345-352.

Clavier J., 1981. - Ecologie descriptive et fonctionnelle du peuplement des sables fins vaseux dans le bassin maritime de La Rance. Thèse Doct. $3^{e}$ cycle. Univ. Paris $6: 232 \mathrm{p}$.

Clavier J., Lechapt J.P., Rétière C. et Rivain V., 1983. Effets à long terme du fonctionnement de l'usine marémotrice sur l'évolution du peuplement des sables fins vaseux de La Rance. Ocean. Acta, Actes du $17^{e}$ Symposium Européen de Biologie Marine, Brest, France: 75-79.

Clavier J. et Richard O., 1985. - Etudes sur les ormeaux dans la région de Saint-Malo. Rapport d'étude A.M.V.L.C.E., $285 \mathrm{p}$.

De Kergariou G., 1971. - L'araignée de mer Maia squinado L. sur le littoral de Bretagne. Sciences et Pêches, 205: 11-19.

Ferns P.N., Hastings M.P. and Shaw T.L., 1984. - Minimizing the possible effects of a tidal power barrage on the shorebird populations of the Severn Estuary. Journal of Environmental Management, $18: 131-143$.
Fischer E., 1929. - Recherches de bionomie et d'océanographie littorales sur La Rance et le littoral de La Manche. Ann. Inst. Océano. Monaco, N.S., 5 (3) : 205-429.

FisCher-PietTE E., 1931. - Sur la pénétration de diverses espèces sessiles dans les estuaires et sa limitation par l'eau douce. Ann. Inst. Océano. Monaco, N.S., 10 (8) : 217-243.

FisCher-PietTE E., 1933. - Nouvelles observations sur l'ordre d'euryhalinité des espèces littorales. Bull. Inst. Océano. Monaco, $619: 1-16$.

Gaillard J.M., 1958. - Haliotis tuberculata Linne. Systématique et distribution. Bull. Lab. Mar. Dinard, 44: 7-11.

Gordon D.C. et Longhurst A.R., 1979. - The environmental aspects of a tidal power project in the upper reaches of the Bay of Fundy. Mar. Poll. Bull., 10: 47-51.

Grall J.R., 1972. - Recherches quantitatives sur la production primaire du phytoplancton dans les parages de Roscoff. Thèse Doct. Etat Univ. Paris : 247 p.

HAN S.J., 1982. - Conditions sédimentologiques comparées pour deux sites d'usine marémotrice: La Baie de Garolim, République de Corée; La Rance, Bretagne, France. Thèse $3^{e}$ cycle, Univ. Paris Sud: 182 p.

Herpe E., 1986. - La végétation algale de La Rance : ses modifications depuis la construction du barrage. Soc. Sc. Bretagne, 58 (1-4), 19-33.

Laboratoire Central d'hydraulique de France, 1982. Etude sédimentologique de La Rance. Rapport général : 21 p.

Lacaze J.C., Le Pemp X. et Villedon de Naide O., 1976. Recherches hydrobiologiques liées à la réalisation de pollutions expérimentales à une grande échelle dans l'estuaire de La 
Rance. Production primaire en relation avec certains paramẻtres physico-chimiques. Bull. Mus. Nat. Hist. Nat., 386 (32) : 71-106.

LANG F., 1986. - Peuplements des fonds durs du bassin maritime de La Rance : rôle fonctionnel de Eupolymnia nebulosa (Annélide Polychète). Doctorat Univ. Rennes I, Fr.: 240 p.

LARSEN P.F. and Topinka J.A. (Edit.), 1984. - Fundy tidal power development. Preliminary evaluations of its environmental consequences to Maine. Technical Report $n^{\circ} 35$, Bigelow Laboratory for Ocean Sciences, West Boothbay Harbor, Maine, U.S.A. : $136 \mathrm{p}$.

Le CALvez J.C., 1986. - Les crustacés décapodes dans le bassin maritime de La Rance; cas particulier du crabe vert Carcinus maenas $\mathrm{L}$. : rôle trophique et impact sur les communautés de substrat meuble. Doctorat Univ. Rennes I, Fr. : 223 p.

LECHAPT J.P., 1983. - Contribution à l'étude des annélides polychètes de substrat meuble en Rance maritime. Thèse $3^{e}$ cycle, Univ. Rennes I, Fr.: 129 p.

Le MaO P., 1985. - Peuplements piscicole et teuthologique du bassin maritime de La Rance : impact de l'aménagement marémoteur. Thèse de Docteur-Ingénieur, E.N.S.A. Rennes, Fr.: $125 \mathrm{p}$.

LE MAO P., 1986. - Feeding relationships between the benthic fauna and the dominant benthic fish of the Rance estuary (France). J. mar. biol. Ass. U.K., 66: 391-401.

Le Mao P., Le Calvez J.C., Lang F. et Fouche M., 1986. Utilisation du bassin de retenue de l'usine marémotrice de $\mathrm{La}$ Rance par les oiseaux aquatiques hivernants. L'Oiseau et R.F.O., 56 (2) : 171-191.

MARIE P., 1938. - Sur la faune des foraminifëres de La Rance. Bull. Lab. Mar. Dinard, 20 : 68-85.

MARTIN W.R., 1970. - Predicted effects of proposed tidal power structures on groundfish in Charlotte County. N.B. J. Fish. Res. Board Canada, 17 (2) : 169-173.

Prıou M.L., 1947. - La répartition des algues dans La Rance. D.E.S., Univ. Rennes : $58 \mathrm{p}$.

RÉTIĖRE C., 1976. - Détermination des classes d'âges des populations de Nephtys hombergii (Annélides Polychètes) par lecture des mâchoires en microscopie électronique à balayage. Structure des populations en Rance maritime. C.R. Acad. Sc. Paris, 282: 1553-1556.

Rétiére C., 1979. - Contribution à la connaissance des peuplements benthiques du golfe normano-breton. Thèse Doct. Etat. Univ. Rennes I, Fr.: 431 p.

RétIĖRE C., 1984. - Energie marémotrice: aspects liés à l'environnement aquatique. La Houille Blanche, 8 : 583-589.

Rétière C. et Richard O., 1980. - Conséquences écologiques de 10 années de fonctionnement de l'usine marémotrice de $\mathrm{La}$ Rance. Rapport E.D.F. - Lab. Mar. Dinard: 71 p.

Rivain V., 1983. - Contribution à l'étude dynamique et fonctionnelle des peuplements de sables fins du golfe normanobreton. Thèse Doct. $3^{e}$ cycle, Univ. Paris VI, Fr. : 166 p.

RouviLlois A., 1967. - Observations morphologiques, sédimentaires et écologiques sur la plage de La Ville-Ger, dans l'estuaire de La Rance. Cahiers Océanographiques, 19 (5) : 375 387.

SHAw T.L. (Edit.), 1980. - An environmental apparaisal of tidal power stations with particular reference to the Severn barrage. Pitman Advanced Publishing Program, Boston, London, Melbourne : $220 \mathrm{p}$.

SChricke V. et Bourgault Y., 1982. - Hivernage des oiseaux d'eau dans l'estuaire de La Rance, 1971-1982. La Sauvagine et sa Chasse, 219: 16-18.

\author{
Adresse de l'auteur \\ Ch. Rétière, \\ Laboratoire maritime, \\ Muséum national \\ d'histoire naturelle, \\ BP 28, 35801 Dinard (France). \\ Tél. : 99461390.
}




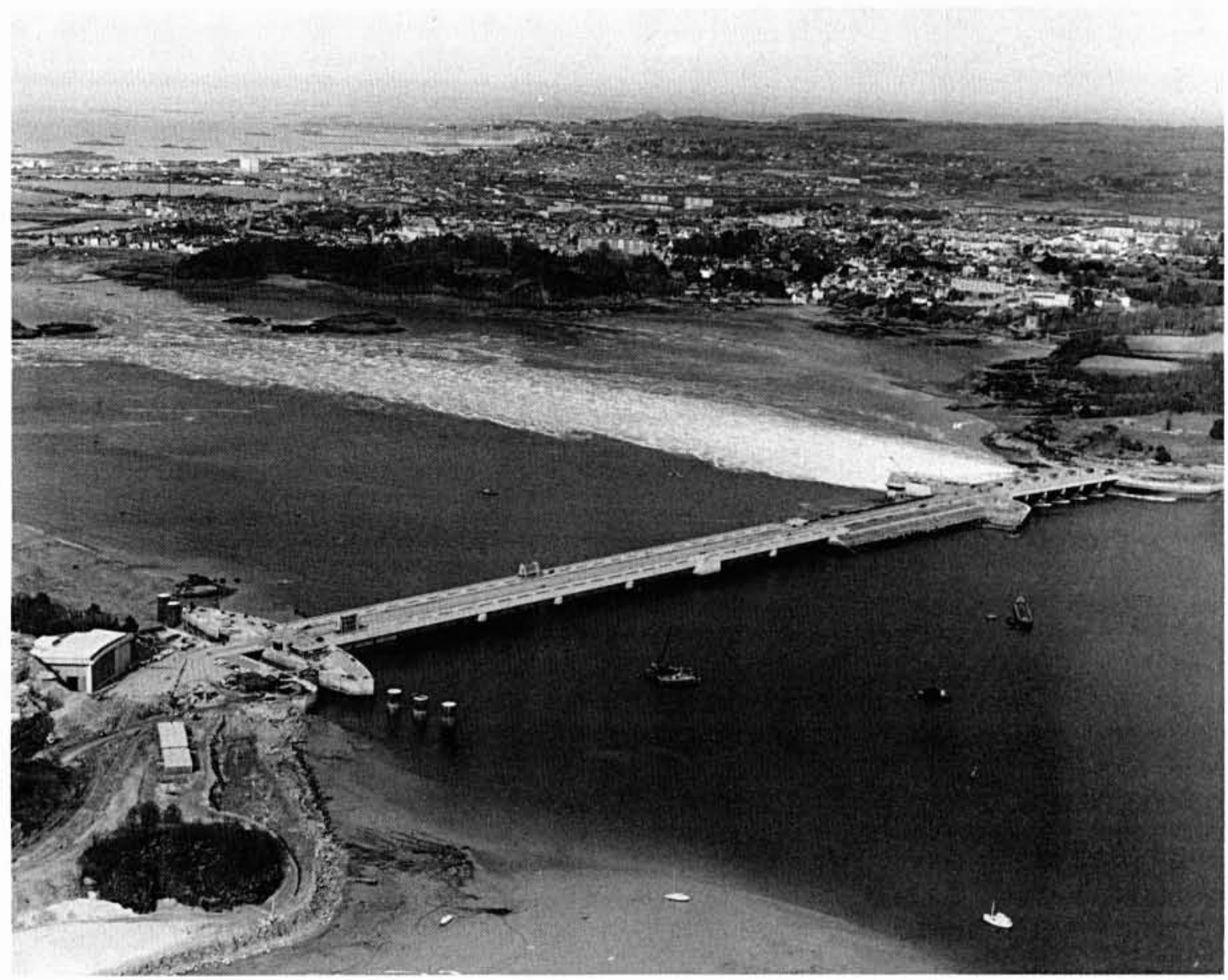




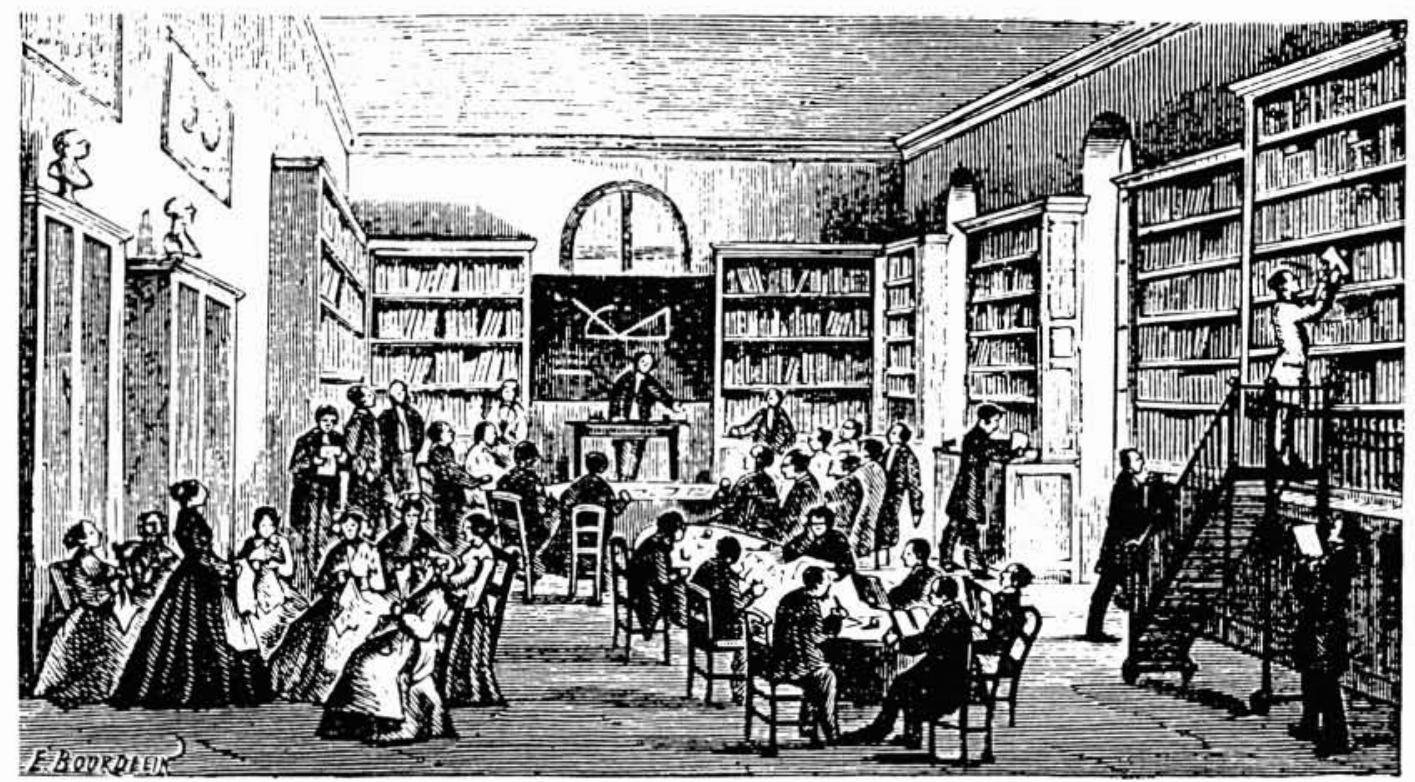

